\title{
Épithètes cultuelles et interprétation philosophique. À propos d'Aphrodite Ourania et Pandemos à Athènes
}

In: L'antiquité classique, Tome 57, 1988. pp. 142-157.

\section{Résumé}

Aphrodite est considérée tantôt comme fille de Zeus et de Dionè, tantôt comme enfant du seul Ouranos. Partant de ces traditions mythiques divergentes, Platon place dans le discours d'un des protagonistes du Banquet une réflexion moralisante sur la dualité de la déesse : Ourania est la divinité céleste, tandis que Pandemos relève d'une conception vulgaire de l'amour. Cette démonstration théorique, à la postérité considérable, ne résiste ni à l'examen des cultes effectifs de la déesse à Athènes ni à l'étude des témoignages parallèles, comme celui de Xénophon.

Citer ce document / Cite this document :

Pirenne-Delforge vinciane. Épithètes cultuelles et interprétation philosophique. À propos d'Aphrodite Ourania et Pandemos à Athènes. In: L'antiquité classique, Tome 57, 1988. pp. 142-157.

doi : 10.3406/antiq.1988.2232

http://www.persee.fr/web/revues/home/prescript/article/antiq_0770-2817_1988_num_57_1_2232 


\section{ÉPITHÈTES CULTUELLES \\ ET INTERPRÉTATION PHILOSOPHIQUE. À PROPOS D'APHRODITE OURANIA ET PANDÉMOS À ATHÈNES}

La divinité, en Grèce classique, se voit attribuer presque autant d'épithètes que d'attributs différents, mais, au-delà de cette multiplication, elle

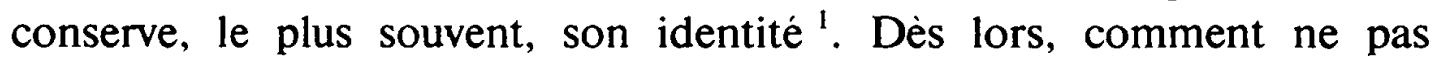
s'étonner de trouver, pour la déesse Aphrodite, l'affirmation répétée d'un partage strict de ses fonctions entre deux épithètes prétendument opposées?

Une tradition tenace, dont nous allons étudier l'origine et l'évolution, a consacré le caractère antithétique des deux épithètes Ourania et Pandémos lorsqu'elles qualifient Aphrodite. Mais il y a lieu de se demander si cette opposition n'est pas purement spéculative et si elle conserve toute sa pertinence quand elle est confrontée à ce que nous pouvons appréhender des cultes effectifs ${ }^{2}$.

\section{LES ORIGINES}

Les premières attestations de la dualité d'Aphrodite se trouvent dans le Banquet de Platon et dans le Banquet de Xénophon, qui semble en être la réplique ${ }^{3}$. Dans l'œuvre de Platon, c'est Pausanias, pédéraste notoire, qui distingue deux Aphrodite, justifiant ainsi l'existence d'un Éros double,

N.B. : les abréviations des titres des revues sont celles de L'Année Philologique.

' J. RUDHARDT, Notions fondamentales et actes constitutifs du culte dans la Grèce classique, Genève, 1958, p. 97.

${ }^{2}$ L. R. FARNELL [ The Cults of the Greek States, II (Oxford, 1896), pp. 660-664] avait déjà posé la question de la lègitimité d'une telle distinction. Nous nous proposons de développer ici la perspective énoncée dans le chapitre qu'il consacre à Aphrodite et qui reste ce qu'il y a de plus complet sur le sujet, même si certains points de vue sont dépassés.

${ }^{3}$ Platon, Banquet, 180d-182a ; Xénophon, Banquet, 8, 9-10. Cf. R. Flaceliére, $\dot{A}$ propos du Banquet de Xénophon, dans REG, 74 (1961), pp. 97-100. 
inséparable de la dualité de sa mère ${ }^{4}$. Voici ce qu'il rapporte à ses convives :

Il y en a une (Aphrodite), la plus ancienne je crois bien, qui, sans avoir eu de mère, est fille d'Ouranos, celle que nous surnommons précisément Ourania ; et la plus jeune, fille de Zeus et de Dionè, que nous appelons, elle, Pandémos 5 .

Dès lors, l'amour n'est plus le dieu univoque présenté par Phèdre dans le discours précédent ${ }^{6}$. L'Éros lié à Aphrodite Pandémos s'adresse plus au corps qu'à l'âme ; il est, comme sa mère, populaire, vulgaire. Il pousse les hommes de basse espèce vers les femmes et les jeunes garçons, affirmant ainsi son rapport avec l'intempérance de la jeune déesse, fille de Zeus et de Dionè ${ }^{7}$. Ourania, par contre, ne possède rien de féminin, c'est-à-dire de corrompu, dans le principe de sa génération ${ }^{8}$ et suscite l'amour désintéressé pour des jeunes gens qui ont dépassé le stade de l'enfance.

Le but de Pausanias est clair. Il s'agit de justifier la pratique de l'amour entre érastes et éromènes au nom de la vertu et de la morale, puisque ce n'est pas la relation pédérastique en soi qui est condamnable, mais les intentions douteuses qui y président parfois ${ }^{9}$.

Dans le Banquet de Xénophon, Socrate en personne prend la parole pour traiter de l'amour, Éros, "cette grande divinité, égale en âge aux dieux éternels, tout en étant la plus jeune d'aspect, maitrisant tout l'univers

${ }^{4}$ Il existe diverses généalogies d'Éros: cf. A. Hermary, art. Eros, dans Lexicon iconographicum mythologiae classicae, III, 1 (1986), p. 850. - La conception de la dualité d'Éros est antérieure à Platon: F. LASSERRE, La figure d'Éros dans la poésie grecque, Lausanne, 1946, pp. 95-103.

${ }^{5}$ Platon, Banquet, 180d-e (trad. d'après L. Robin) : $\dot{\eta} \mu \varepsilon \dot{v} v$ $\gamma \dot{\varepsilon} \pi o v \pi \rho \varepsilon \sigma \beta v \tau \varepsilon \rho a \varkappa \alpha i$

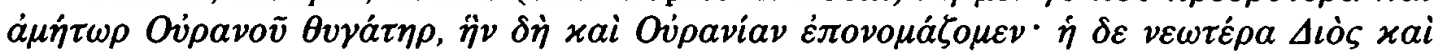

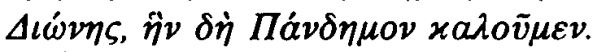

- Platon, Banquet, 178a-180b.

7 Deux générations séparent Zeus et Ouranos, les "pères respectifs".

${ }^{8}$ Cf. HÉsIOdE, Théogonie, 190-206 : Aphrodite est née de l'écume émergeant du sexe d'Ouranos tranché par Cronos et tombé dans la mer.

${ }^{9}$ Platon, Banquet, 181a : «... ce n'est pas pour tout amour qu'on dira : 'il est beau, il est digne qu'on en célèbre les louanges', mais pour celui-là seul de qui est belle l'impulsion à aimer" (trad. L. Robin). Sur la pédérastie dans l'antiquité, voir K J. Dover, Greek Homosexuality, Harvard Univ. Press, 1978 ; F. BufFIERE, Éros adolescent. La pédérastie dans la Grèce antique, Paris, 1980 ; J. MAZEL, Les métamorphoses d'Éros. L'amour dans la Grèce antique. Paris, 1984, pp. 133-170. 
par sa puissance, mais établie dans le cœur des hommes» ${ }^{10}$. Et il en vient tout naturellement à poser le problème d'Aphrodite :

Existe-t-il une seule Aphrodite ou bien deux, Ourania et Pandemos, je ne sais, car Zeus, qui parait toujours le mème, possède de nombreux noms. Ce que je sais, pourtant, c'est que, pour chacune des deux séparément, il existe des autels et des temples, et aussi des rites qui, pour la Pandémos, sont pleins de relâchement, tandis qu'ils sont plus purs pour Ourania. On peut conjecturer que la Pandémos nous fait aimer les corps, Ourania l'âme, l'amitie et les belles actions ".

À présent, comparons sommairement les données principales des textes. Dans celui de Platon, le discours supporté par des arguments mythologiques attribue à Pandémos l'amour hétérosexuel, ainsi que le penchant répréhensible pour les tout jeunes garçons. Ourania, quant à elle, préside à l'amour masculin dans sa forme la plus désintéressée. Chez Xènophon, la césure instaurée par Socrate entre les deux épithètes est plus nette ; Aphrodite Pandémos suscite en nous le désir des corps alors que Ourania nous insuffle l'amour de l'âme, que ce soit celle de l'homme ou celle de la femme. En ce qui concerne la dualité de la déesse, il est important de souligner la prudence que Xénophon attribue aux propos de Socrate, qui dit ne pas savoir (oủx oí $\delta$ ) s'il y a deux déesses. Le philosophe semble ennuyé, et l'exemple de Zeus, unique malgré la pluralité de ses noms, illustre son hésitation en la justifiant. Il poursuit malgré tout en invoquant une certitude (ol $\delta \alpha$ ) basée sur le rituel; nous y reviendrons.

Cette tradition, que l'on qualifiera de "philosophique» pour faire bref, utilise donc la mythologie et les rites en vue d'étayer une réflexion morale. Cette spéculation érudite ne semble cependant pas être la seule origine possible de la conception d'une Aphrodite double. La cité d'Athènes connait, en effet, un culte d'Aphrodite Pandémos situé sur le flanc sud-ouest de l'Acropole et dont la fondation est, entre autres, attribuée à

${ }^{10}$ Xenophon, Banquel, 8, 1 (trad. F. Ollier).

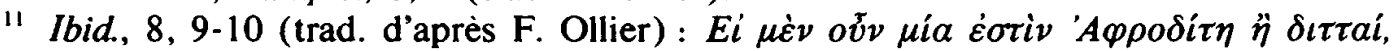

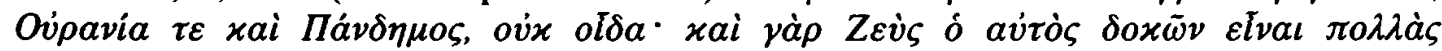

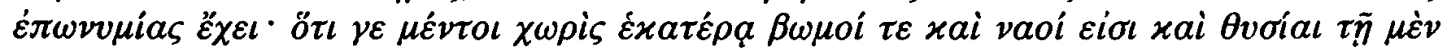

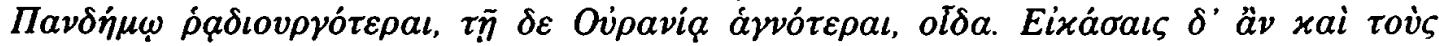

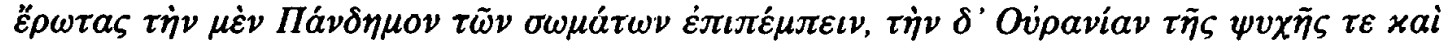

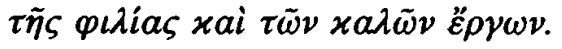


Solon ${ }^{12}$. Le contexte de cet établissement est assez particulier. L'archonte athénien aurait voulu remédier aux inconvénients que procurait à la cité la fougue d'une jeunesse difficilement maitrisable. Il aurait pour ce faire établi des esclaves féminines dans des maisons spécialisées et, avec l'argent amassé par les tenancières, aurait institué le culte d'Aphrodite Pandémos. Nicandre de Colophon, l'auteur cité par Athénée comme source de son information, vivait au II $^{\mathrm{e}}$ siècle avant J.-C. et, deux siècles avant lui, Philémon avait déjà parlé de la fondation des maisons closes par Solon sans lui attacher celle du culte ${ }^{13}$.

Il semble bien qu'une telle tradition peut être rapprochée de la définition d'Aphrodite Pandémos que l'on trouve dans le discours philosophique athénien du Iv ${ }^{e}$ siècle. Reste à savoir, et l'on ne peut que poser la question, si la tradition populaire est antérieure ou non à l'interprétation du Banquet. Quoi qu'il en soit, la conception philosophique et le récit de Nicandre appartiennent à une veine similaire, soit que l'un ait influencé l'autre, soit que les deux traditions aient, chacune indépendamment ${ }^{14}$, contribué à l'élaboration du partage des fonctions de la déesse et au succès littéraire qui en a découlé.

\section{LA POSTÉRITÉ}

Avant d'étudier les cultes tels qu'ils étaient pratiqués à Athènes, il convient de s'interroger sur la postérité d'un tel partage pour en saisir toutes les implications. On retrouve, en effet, la marque très nette des deux traditions évoquées ci-dessus. L'interprétation populaire qui érige Pandémos en patronne des hétaïres a par la même occasion consacré Ourania comme protectrice des unions légales. Par exemple, au III $^{\mathrm{e}}$ siècle avant J.-C., une épigramme de Théocrite, censée se trouver sur une statue

12 Nicandre de Colophon, cité par Athenee, Banquet des Sophistes, XIII, 569d-e et

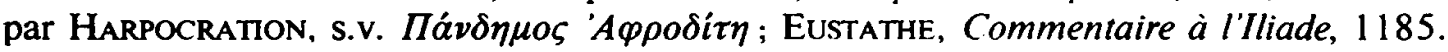
- Pour la localisation, voir L. BESCHI, Contributi di topografia ateniese, dans $A S A A, 45-46$ (1967-1968), pp. 517-528 ; G. DonTAS, The True Aglaurion, dans Hesperia, 52 (1983), pp. 48-63.

${ }^{13}$ Phil Emon, frg. 4 Kock ( $C A F$, II, p. 479$)=$ frg. 4 Edmonds (The Fragments of Attic Comedy, IIIA, p. 6), cité par ATHENEE, XIII, 569d. - Sur une hypothétique localisation des maisons closes, voir l'article de C. N. Edmonson, The Leokoreion in Athens, dans Mnemosyne, 17 (1964), pp. 375-378.

${ }^{14}$ M. le professeur A. Motte me signale cependant qu'il serait étonnant que Platon, bon connaisseur des cultes et des traditions religieuses athéniennes, n'ait pas exploité la version populaire, si elle existait précédemment. 
d'Aphrodite, précise que "cette Aphrodite n'est pas Pandémos" et recommande d'invoquer la déesse en l'appelant Ourania. "Elle a été consacrée par la chaste Chrysogone dans la demeure d'Amphiclès, le père de ses enfants, le compagnon de sa vie» ${ }^{15}$. Cette distinction entre l'amour conjugal et les relations illicites se retrouve dans l'Interprétation des songes d'Artemidore d'Éphèse, au II $^{\mathrm{e}}$ siècle de notre ère. D'après lui, un rêve mettant en scène Aphrodite Pandémos est bon pour les courtisanes, mais prédit honte et blâme à la ménagère et aux futurs époux. Aphrodite Ourania est, pour sa part, "favorable aux mariages, aux relations sociales et à la naissance des enfants. Elle est, en effet, la cause des unions et des descendances. Elle est également favorable aux paysans, car elle est considéree comme nature et mère de l'univers" ${ }^{16}$.

D'autre part, les Banquet(s) du Iv $v^{e}$ siècle ont consacré la division entre l'amour désincarné suscité par Aphrodite Ourania et celui des sens insufflé par Pandemos. Divers exemples, liés directement ou de façon plus detournee à la tradition platonicienne, illustrent cette perspective qui est attestee jusqu'aux époques les plus basses. Ainsi Lucien de Samosate distingue, par l'intermédiaire de personnages dialoguant sur l'amour, l'Éros ouranien, divinité vénérable, de l'autre Éros, insensé et puéril. Le premier permet de goûter la volupté mêlée à la vertu, tandis que le second fait du désir de la femme son principal souci ${ }^{17}$. Cet Éros double ( $\delta \iota \pi \lambda o \tilde{v} \varsigma$ $\theta \varepsilon \dot{s} \varsigma \delta^{\prime}$ "E $\omega \omega \varsigma$ ) n'est pas ici associè à sa mère, mais la réflexion s'inscrit manifestement dans la tradition imaginée par Platon lorsque nous retrouvons la différence entre plaisir sans mesure ( $\tau \dot{\eta} v \dot{\alpha} \varkappa o ́ \lambda a \sigma \tau o v \dot{\eta} \delta o v \dot{\eta} v$ )

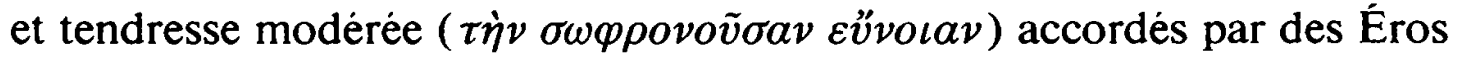
d'âge différent ${ }^{18}$. Par ailleurs, le scholiaste d'une autre œuvre de Lucien définit l'épithète Pandémos comme une qualification «de l'Aphrodite honteuse»" ${ }^{19}$.

Dans le cadre du culte, il arrive parfois que des statues d'Aphrodite Pandémos et d'Ourania coexistent à l'intérieur d'un même temple.

is Théocrite, Épigrammes, 13 (= Anth. Pal.. VI, 340) (trad. d'après Ph.-E. Legrand) :

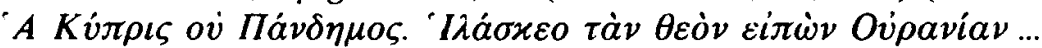

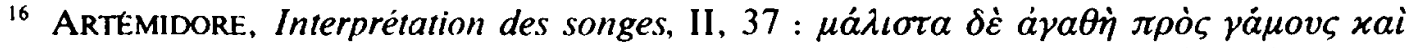

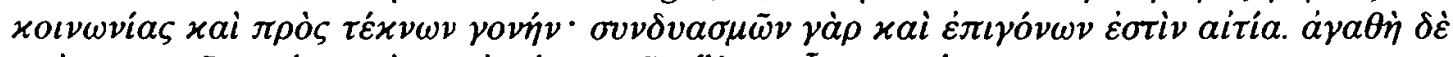

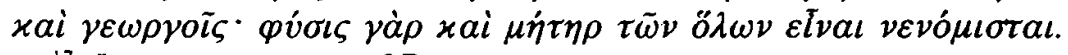

17 LUCIEN, Amours, 37.

18 Rappelons que le facteur d'âge se trouvait dejà chez Platon (Bunquet, 180d-e).

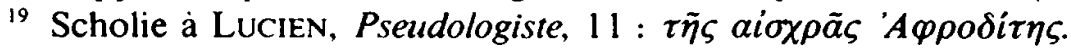


Pausanias nous a conservé, dans sa Périégèse, le souvenir d'une telle cohabitation. Pour Élis et Mégalopolis d'Arcadie où le cas est attesté ${ }^{20}$, il n'émet aucun jugement sur la valeur respective des épithètes, mais, à Thèbes, devant les statues élevées par Harmonie, il distingue Ourania qui préside «à l'amour pur, libre du désir des corps» et Pandémos, celle qui favorise «les relations sexuelles» ${ }^{21}$.

À l'intérieur d'une postérite plus philosophique, les exemples abondent ${ }^{22}$. Un des plus éclairants est tiré des discours d'Himérios ( $\mathrm{IV}^{\mathrm{e}}$ siècle après J.-C.) dont des extraits sont conservés dans la Bibliothèque de Photios. On y retrouve un écho de l'amour pédérastique :

Aphrodite Pandémos n'a rien de commun avec Ourania. La première, en effet, engendre les amours vulgaires et dont la nature n'est pas épurée, mais l'autre, ses enfants sont en or et d'or leurs traits, et les cibles que frappent ces traits sont des âmes jeunes et intactes ${ }^{23}$.

Force est donc de constater que les deux interpretations se sont perpétuées, sans manquer de se rejoindre parfois ${ }^{24}$. Aujourd'hui encore, on retrouve des appréciations du même type que celles de l'Antiquité. Par exemple, dans l'édition des épigrammes de l'Anthologie Palatine dans la Collection des Universités de France, P. Waltz estime que l'épigramme de Dioscoride (III ${ }^{e}$ s. av. J.-C.) par laquelle une hétaïre consacre un éventail

20 PaUs., VI, 25, 1 ; VIII, 32, 2.

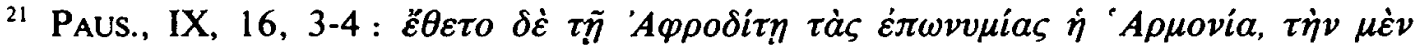

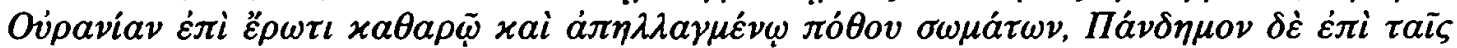
$\mu i \xi \varepsilon \sigma l$.

22 JEAN LE Lydien, De mensibus, IV, 64 : il reprend simplement la tradition Plutarque, Moralia, 764b : il parle des deux amours connus des Égyptiens : $\tau o ́ v \tau \varepsilon$

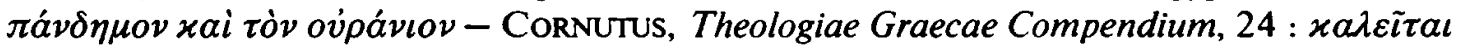
(í 'A

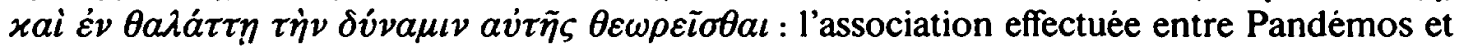
la terre repose sans doute sur le pouvoir procreateur qui caractérise la sphère féminine à laquelle appartiennent Pandémos et Gê. Voir à ce propos un autre passage de Platon (Banquet, 190b) ; Julien (IMP.), Convivium (Caesares), 31 la, et l'explication que J. F. Gilluam donne du passage [Titus in Julian's Caesares, dans AJPh, 83 (1967), pp. 203-208].

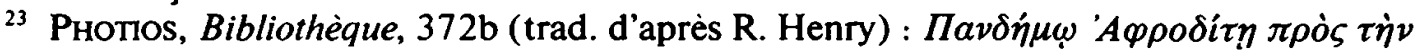

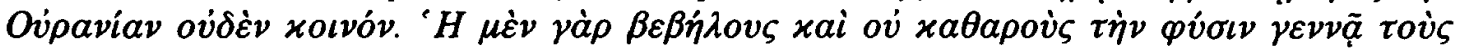

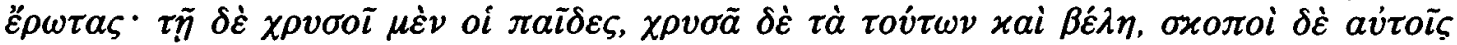

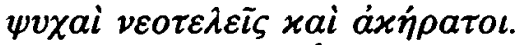

${ }_{24}$ Hellodore, Éthiopiques, I, 19, 7. Cf. E. Feulllátre, Études sur les Éthiopiques d'Héliodore, Paris, 1966, p. 149. 
à Ourania «comme dîme des gains de son lit» n'est qu'une «effronterie de fille légère»" ${ }^{25}$.

Il existe, parallèlement, des indices fournis par le culte invitant à considérer une autre conception de la divinité.

\section{Ourania et Pandémos À Athènes}

Si nous envisageons le cas d'Athènes, c'est pour deux raisons principales. En premier lieu, la cité a accueilli la réflexion philosophique dont nous sommes partis. Or les textes font référence à des données cultuelles qui, même si elles ne possèdent qu'une valeur d'exemple, se rattachaient peut-être à une réalité locale. Ensuite, les cultes d'Aphrodite sont bien

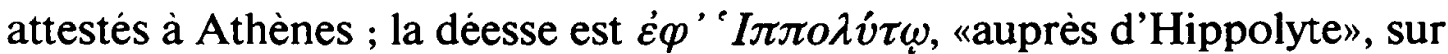
le flanc sud de l'Acropole ${ }^{26}$, tandis qu'elle est associée à Éros au flanc nord et semble porter l'épithète $\dot{\varepsilon} \nu \varkappa \dot{\eta} \pi \circ \iota \varsigma$, dans des jardins ${ }^{27}$. La même dénomination se retrouve dans le sanctuaire qui lui est consacré au bord de l'Ilissos ${ }^{28}$. Ces certitudes mises à part, il existe d'autres épithètes dont la réalité paraît moins affirmée. Aphrodite est yívoos chez certains lexicographes qui l'associent à Éros et à Hermès du même nom ${ }^{29}$. Elle est peut-être $\beta \lambda a \dot{v} \tau \eta$, «déesse à la sandale», en contre-bas du bastion d'Athéna Nikè, près des Propylées ${ }^{30}$. On connait également une Aphrodite $\dot{\varepsilon} \pi \imath \tau \rho \alpha \gamma i a$, sans savoir si elle fait l'objet d'un culte indépendant ${ }^{31}$.

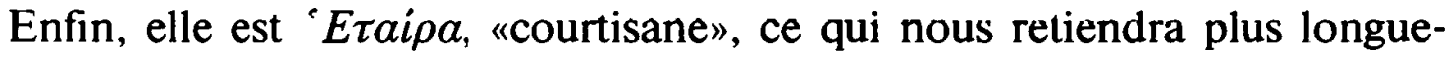

25 P. WALTZ, Anthologie Palatine, épigrammes votives, VI, $\mathrm{n}^{\circ} 290$, Paris, 1931 (Coll. des Universités de France), p. 145, n. 1. Dans l'édition de A. S. F. Gow et D. L. Page (Hellenistic Epigrams, I, Cambridge, 1965, p. 245), le commentaire est beaucoup plus mesuré, d'autant que d'autres épigrammes reflètent l'existence de telles dédicaces (Anth. Pal., VI, 206, 207) qui donnent à penser que notre hétaïre espère trouver un mari !

${ }^{26}$ Euripide, Hippolyte, 29-33 ; Ascléplade de Tragilos, 12 F 28 J. ; Diodore, IV, 62, 2 ; TZETZÈs, schol. à LyCOPHRON, Alexandra, 1329 ; I.G., I' ${ }^{2}, 324,1.69 ; 310,1.280$.

${ }_{27}$ Paus., I, 27, 3. Cf. O. Broneer, Eros and Aphrodite on the Athenian Slope of the Acropolis in Athens, dans Hesperia, 1 (1932), pp. 31-55; dans Hesperia, 2 (1933), pp. 329-417; 4 (1935), pp. 109-188.

${ }_{28}$ Paus., I, 19, 2 ; Pline, Histoire naturelle, XXXVI, 16 ; Lucien, Portraits, 4 et 6 ; I.G., $\mathrm{II}^{2}, 324,1.85$.

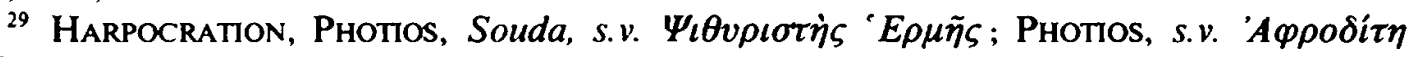
wívoos; Anecdota Graeca, p. 317, 11 Bekker.

${ }^{30}$ I.G., $\mathrm{II}^{2}, 5185$. Voir à ce propos mon article Aspects orientaux du culte d'Aphrodite à Athènes, dans Studia Phoenicia, 5 (1987), pp. 153-155.

${ }^{31}$ I.G., $\mathrm{II}^{2}, 5115,5148$. 
ment dans la suite ${ }^{32}$. Un tel passage en revue, obligatoirement bref, montre déjà combien la division en deux entités est arbitraire.

\section{a) Ourania}

La déesse des philosophes, fille d'Ouranos, préside à l'amour "pur» qui, selon une autre tradition, sera confondu avec l'amour légal. Prenons donc à témoin deux textes pour montrer que cette affirmation ne s'accorde pas nécessairement aux données du culte. Le premier nous est déjà connu puisqu'il s'agit de l'épigramme de Dioscoride où une hétaïre consacre un éventail à Ourania ${ }^{33}$. Le second est extrait des Dialogues des Courtisanes de Lucien où une hétaïre athénienne parle d'offrir une génisse à Ourania et une chèvre à Pandémos ${ }^{34}$. Elle adresse donc autant ses prières à l'une qu'à l'autre, même si les offrandes ne sont pas d'égale valeur ${ }^{35}$. Évidemment, ce genre de littérature doit être utilisé prudemment. Les buts poursuivis, tant par l'épigramme que par les Dialogues, ne permettent pas d'en déduire un constat religieux ou cultuel pur et simple. Cependant, pour que la satire soit efficace, les hétaïres présentées doivent avoir correspondu à une réalité. Et il semble qu'Ourania a pu aider les courtisanes dans la quête d'un mari ${ }^{36}$, ce qui ne la confine pas nécessairement dans la sphère des "honnêtes femmes». Signalons enfin que le seul culte d'Aphrodite auquel étaient soi-disant associées des hiérodules en Grèce est celui d'Ourania à Corinthe ${ }^{37}$.

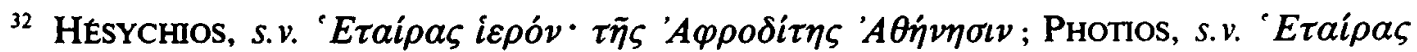

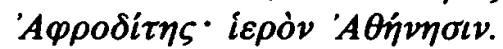

${ }^{33}$ Cf. supra, n. 25.

${ }^{34}$ LuCIEN, Dialogues des Courtisanes, 7, 1.

${ }^{35}$ Notons au passage la scholie qui commente cet extrait et s'inscrit dans la tradition

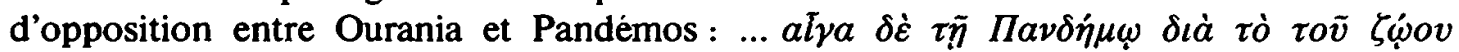

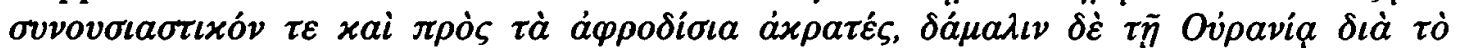

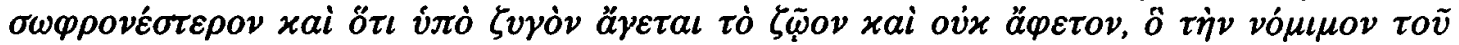

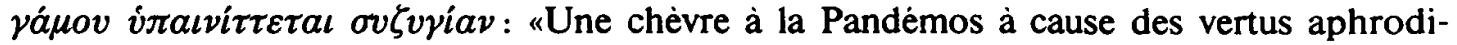
siaques de l'animal et aussi de son intempérance sexuelle, une génisse à Ourania à cause de sa plus grande modération et parce que cet animal se déplace sous le joug et ne peut paitre librement, ce qui fait allusion à l'union habituelle dans le mariage».

${ }^{36}$ Cf. supra, n. 25.

37 Pindare, frg. 122 Bergk. Cf. L. R. Farnell, o.l., p. 668. - La réalité de la prostitution sacrée à Corinthe est sérieusement mise en doute par H. D. SAFFrEY, Aphrodite à Corinthe. Réflexions sur une idée reçue, dans $R B i, 92$ (1985), pp. 359-374. Quoi qu'il en soit, les prostituées, sacrées ou profanes, étaient directement liées au nom de la déesse de l'Acrocorinthe. 
Un autre exemple peut être intégré à la démonstration, mais il est plus hypothétique. Des lexicographes nous ont conservé le souvenir d'une deesse Hètaïra honoree par les Athéniens ${ }^{38}$. Apollodore d'Athènes, qui pourrait être la source des auteurs précédents, affirme que "Hètaira est l'Aphrodite qui rassemble les amis et les amies ${ }^{39}$ " et Clément d'Alexandrie critique, quelques siècles plus tard, les Athéniens qui offrent des sacrifices à Aphrodite Hétaira ${ }^{40}$. Étant donné que cette Courtisane divinisee n'est pas attestée qu'à Athènes ${ }^{41}$, on peut légitimement supposer que l'épithète recouvrait une réalite autre que litteraire ou apologétique. Sans aller jusqu'à pretendre qu'Hétaira est un nom de culte d'Aphrodite à Athènes, il est possible de le rapprocher d'un culte tout à fait officiel dans la cité.

Athènes connaissait deux cultes d'Aphrodite Ourania, un sur l'agora ${ }^{42}$, et un autre, un peu particulier, dans le mème téménos qu'Aphrodite $\dot{\varepsilon} v$ xं́лoıৎ au bord de l'Ilissos ${ }^{43}$. Le caractère végetal de la déesse en ce lieu semble suffisamment mis en évidence par l'épithète pour que l'on n'ait pas à s'appesantir sur la question. Or des courtisanes athéniennes qui accompagnaient l'armée de Périclès pendant la guerre samienne de 440/439 ont fondé, à Samos, un culte d'Aphrodite $\dot{\varepsilon} v$ É $\lambda \varepsilon \iota$ ou $\dot{\varepsilon} \nu \varkappa \alpha \lambda \dot{\alpha} \mu o \iota \varsigma^{44}$. Il est évident que la réalité physique de l'endroit a déterminé l'attribution d'une telle épithète, tout comme les bords de l'llissos ont marqué l'Aphrodite qui y est honoree. 11 est dès lors tentant de reconnaitre dans le culte $\dot{\varepsilon} \nu$

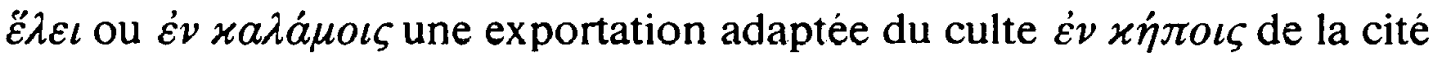
natale des hétaïres. Si l'on accepte l'hypothèse, il devient possible d'étu-

${ }^{38}$ Cf. supra, n. 32.

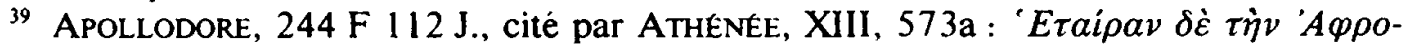

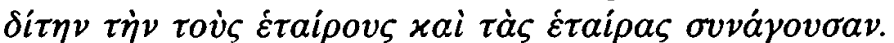

40 Clément d'AleXandrie, Protreptique, II, 39, 2.

${ }^{41}$ Hétaira à Éphèse : EUAlCÈs, $418 \mathrm{~F} 2 \mathrm{~J}$., cité par ATHENÉE, XIII, 573a ; sanctuaires d'Hétaira partout en Gréce : PhilÉtaIRe, frg. 8 Kock $(C A F$, II, p. 232) $=$ frg. 5 Edmonds (o.l., II, p. 22), cité par ATHENÉ, XIII, 572d.

${ }^{42}$ Paus., I, 14, 7. Pour la localisation. voir T. L. Shear, dans Hesperia, 53 (1984), pp. 24-40.

${ }^{43}$ Paus., I, 19, 2 : un pilier hermaïque à tête féminine où il est indiqué qu' "Aphrodite Ourania est la plus àgee de celles que l'on appelle les Moires». - Sur cette forme particulière de représentation, voir mon article l.l., dans Studia Phoenicia, 5 (1987).

${ }_{44}$ AleXIs DE SAMOS, $539 \mathrm{~F} 1 \mathrm{~J}$., cite par ATHENEE, XIII, $572 \mathrm{f}$. L'hypothèse qui suit avait dejà èté emise par W. H. ENGEL, Kypros, II (1841), p. 378, et fut reprise par H. METZGER, Un lébès gamikos à figures rouges du Musée National d'Athènes, dans $B C H, 66-67$ $(1942-1943)$, p. 243 , pour interpréter le groupe de dariseuses représenté sur le vase en question. 
dier plus précisément la dénomination 'Eqaípa attribuée à Aphrodite. En effet, les courtisanes forment une catégorie particulière de fidèles de la déesse $\dot{\varepsilon} v x \dot{\eta} \pi 0 \iota \varsigma$ dont la nature ouranienne a été démontrée ailleurs ${ }^{45}$. Peut-être, au vu de tous ces éléments, Hétaira était-elle une appellation populaire de l'Aphrodite $\dot{\varepsilon} v \varkappa \dot{\eta} \pi o \iota s$, à la suite de la dévotion privilégiée des courtisanes à son égard. Ourania, dont une représentation hermaïque se trouvait dans le téménos de l'Ilissos, ne semblait donc pas inconciliable, par une sorte de vertu particulière, avec un tel culte. Et si l'on prend le témoignage de Lucien en considération, on voit que c'est à Ov́paviáa ćv $\varkappa \dot{\pi} \pi o \iota \varsigma$ que la courtisane abandonnée adresse ses prières. Ainsi la pure déesse des philosophes peut aussi bien recevoir un culte en tant que déesse des courtisanes.

\section{b) Pandémos}

Les jeux de mots peuvent avoir des conséquences importantes. Il semble, en effet, que la polysémie du mot $\delta \tilde{\eta} \mu o \varsigma$ n'est pas étrangère à la conception que nous avons présentee. Car $\delta \tilde{\eta} \mu o \zeta$ est autant populus que plebs $^{46}$. Les deux traditions attachées à la fondation du culte d'Aphrodite Pandémos à Athènes sont directement liées à cette ambiguïté. Nous avons déjà parlé de celle qui met en relation Solon et les maisons closes. Le sens de «bas peuple» a seul été pris en ligne de compte. L'autre version a été recueillie par Pausanias, qui visitait le temple de la déesse. La fondation en est, cette fois, attribuée à Thésee et est considérée comme une conséquence de la réunion des Athéniens des dèmes en une seule cité ${ }^{47}$. Aphrodite est la déesse du peuple entier, le peuple souverain trouve en elle une protectrice attentive ${ }^{48}$.

Ce préambule permet de préciser les deux aspects du problème : Aphrodite Pandémos est-elle la jeune désse depravée évoquée chez Platon ou, au contraire, la caution religieuse d'un acte politique et la protectrice du corps civique ? Il y a un siècle, certains savants ont accordé beaucoup de crédit à la tradition platonicienne et à son pendant populaire

${ }^{45}$ Cf. supra, n. 43.

${ }^{46}$ LIDDELL-SCoTt, s.v. $\delta \tilde{\eta} \mu o \zeta$. Il semble même que le mot a pu signifier "fille publique" chez Archiloque (frg. 184 Bergk).

${ }^{47}$ Paus., I, 22, 3.

48 Parmi les nombreux sens du mot $\delta \tilde{\eta} \mu o \varsigma$, on trouve également la notion de "deme". Même si l'épithète $\pi a ́ v \delta \eta \mu o \zeta$ reprend le mot au singulier, peut-être cette connotation particulière était-elle aussi suggérée dans le contexte du synœcisme de Thésée. 
de la fondation du culte par Solon, allant jusqu'à refuser toute crédibilité à Pausanias et donc l'existence même d'un culte athénien à Pandèmos ${ }^{49}$. Il ne pouvait s'agir que de l'appellation populaire d'Aphrodite $\dot{\varepsilon} \varphi$ ' 'I $\pi \pi 0$ $\lambda v i \omega$ que Pausanias ne nomme pas dans sa description du flanc sud de l'Acropole, mais connue par ailleurs à cet endroit ${ }^{50}$. Cependant, il a bien fallu reconnaitre le manque de validité de cette hypothèse après la découverte d'une inscription on ne peut plus officielle concernant le culte d'Aphrodite Pandemos ${ }^{51}$.

Il s'agit d'un décret de l'année 284 avant J.-C. ordonnant des dépenses

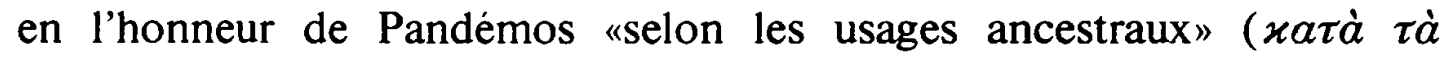
$\pi a ́ \tau \rho \iota a)$. Il est recommandé aux astynomes de prendre soin du sanctuaire et, pour ce faire, de disposer d'une colombe pour la purification, de faire nettoyer les autels, d'enduire les portes de poix et de laver les statues (la fin de l'inscription est lacunaire) lors de la procession pour Aphrodite Pandémos. Le texte mentionne également la prêtresse de la déesse, dont un parent dit être présent lors de la prise de décision. Nous savons par ailleurs que, au $\mathrm{IV}^{\mathrm{e}}$ siècle, la charge avait été attribuée à une citoyenne athénienne, dont la famille a tenu à honorer la deesse et s'adresse à elle

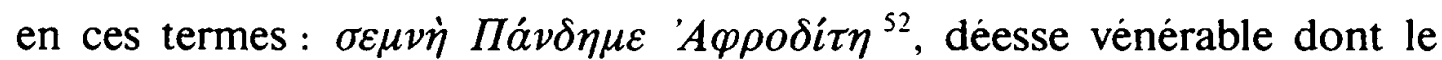
culte semble remonter au $\mathrm{Vl}^{\mathrm{e}}$ siècle au moins ${ }^{53}$.

Se basant probablement sur une tradition semblable à celle dont Pausanias a tiré ses informations, Apollodore d'Athènes justifie la situation du culte d'Aphrodite Pandemos près de l'ancienne agora par le fait que, "autrefois, tout le peuple se réunissait aux assemblées que l'on

49 U. Koenler, dans $M D A I(A), 2$ (1877), pp. 175-176 ; J. G. Frazer, Pausanias' Description of Greece, II (1898), pp. 243-246; A. W. VERRALl, Aphrodite Pandemos and the Hippolytus of Euripides, dans $C R, 15$ (1901), p. 449 ; A. N. OIKoNOMides, The Two Agoras in Ancient Athens, Chicago, 1964, p. 6.

so Cf. supra, n. 26. Cf. L. BESCHI, l.l., pp. 523-525, avec, p. 517, une hypothèse visant à expliquer le silence de Pausanias.

s1 I.G. $\mathrm{II}^{2}, 659$ = F. SokolowSKI, Lois sacrées des cités grecques, Paris, 1969, n 39.

s2 $I . G$. II $^{2}, 4596$. Il s'agit d'une dédicace inscrite sur un fragment de l'épistyle du petit naos d'Aphrodite Pandèmos. On y apprend que l'offrande ètait faite par la prêtresse, sa sœur et le fils de celle-ci.

${ }^{53}$ Erika Simon, Aphrodite Pandemos auf attischen Münzen, dans Schweizerische Numismatische Rundschau, 49 (1970), pp. 5-24. L'auteur analyse un tétradrachme du temps de Clisthène, qui reprèsente Athena d'un côté et une tête féminine bifrons de l'autre. E. Simon démontre de façon convaincante qu'il peut s'agir d'Aphrodite Pandemos et de Peitho honorées ensemble sur le flanc sud de l'Acropole. 
appelait agora" ${ }^{54}$. Même s'il est certain aujourd'hui que le renseignement topographique est incorrect, la connotation politique est intéressante. Ce caractère particulier d'Aphrodite est attesté aussi bien en Grèce continentale qu'en Asie Mineure et dans les îles où elle protège soit l'ensemble $\mathrm{du}$ corps civique, soit une magistrature précise ${ }^{55}$. A Athènes, outre

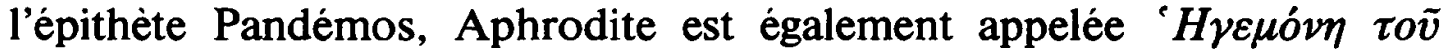
$\delta \dot{\eta} \mu o v$ en association avec les Charites dans une dédicace effectué par la Boulè (fin III ${ }^{e}$ siècle avant J.-C.) et inscrite sur un autel retrouvé in situ en bordure de la Voie sacrée, près du temple d'Ourania ${ }^{56}$. Aphrodite et les Grâces sont porteuses de valeurs très proches, la vie végétale, le cycle de la reproduction et l'harmonie entre les hommes ${ }^{57}$. Ce dernier aspect est ici tout particulièrement mis en évidence et incite à effectuer certains rapprochements qui, même s'ils sont issus de traditions assez tardives, révèlent une conception religieuse.

Quand Solon se voit attribuer la paternité du culte d'Aphrodite Pandémos, un courant étiologique s'en sert pour légitimer la prostitution. Mais Solon est avant tout le grand réformateur du début du vi ${ }^{e}$ siècle, celui qui a tenté, même modérément, de réduire les oppositions entre factions ${ }^{58}$. Tout comme Thésée, quelques siècles légendaires plus tôt, avait réuni les Athéniens en une cité, usant d'une persuasion fructueuse plutôt que d'une violence inutile ${ }^{59}$. L'association de des deux noms avec

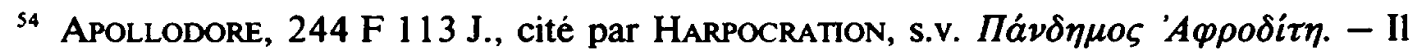
semble qu'Apollodore est aussi la source de la Souda qui, sous le même intitulé, rapporte des faits identiques. Cf. G. Dontas, l.l., p. 63.

ss J. et L. ROBERT, dans REG, 72 (1959), pp. 229-230, n 325 ; REG, 77 (1964), pp. 144-145, $\mathrm{n}^{\circ} 82$; ibid., pp. 193-194, n 288 ; F. Croissant-F. Salviat, Aphrodite gardienne des magistrats : gynéconomes de Thasos et polémarques de Thèbes, dans $B C H$, 90 (1960), pp. 460-471 ; F. SoKolowsKI, Aphrodite as guardian of Greek magistrates, dans $H T h R, 57$ (1964), pp. 1-8. - Zeus remplit également cette fonction, en Attique, sous le nom de Pandémos : I.G., II $^{2}, 1075,1.19$ (époque d'Hadrien).

s6 I.G., II ${ }^{2}, 2798$. G. WELTER, Datierte Altäre in Athen, dans $A A, 1939$, pp. $35-36$; J. Travlos, Pictorial Dictionary of Ancient Athens, 1971, p. 80, fig. 102.

57 A. Furtwaengler-H. W. Stoll, art. Charis-Chariten, dans W. H. Roscher, Lexikon ..., I (1884-1886), col. 873-884 ; Maria RocchI, Contributi allo culto delle Charites, II, dans StudClas, 19 (1980), pp. 19-28. - Cf. la thèse très intéressante de J. H. Oliver, Demokratia, the Gods and the Free World, Baltimore, 1960, pp. 91-118.

${ }^{58}$ Parmi l'abondante bibliographie sur le sujet : J. LABARBE, Les premières démocraties de la Grèce antique, dans Bull. de l'Acad. R. de Belg., Cl. des Lettres et des Sc. mor. et polit., 58 (1972), pp. 228-230.

s9 Plut., Thésée, 24, 2 ; Pausanias (I, 22, 3) nous apprend que Peitho ètait honorée aux côtés d'Aphrodite Pandémos. 
Pandémos ne peut relever d'une coïncidence. Malgré la différence des personnes, la perspective envisagée est la même.

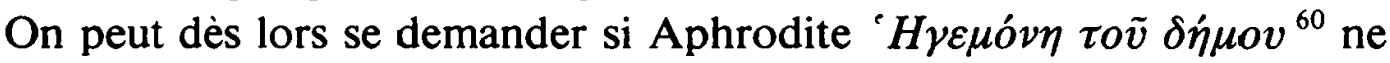
pourrait pas être une sorte d'émanation de la Pandémos, située sur le flanc de l'Acropole et donc de façon moins centrale par rapport au cœur politique qu'est l'agora du III $^{e}$ siècle ${ }^{61}$. La "Conductrice du peuple» constituerait ainsi un rappel de la déesse du "Peuple entier», sur l'agora, près du temple d'Ourania. Nous sommes encore une fois bien loin des considérations théoriques du Banquet, auquel nous allons revenir un moment avant de clore la démonstration.

\section{4. $\Theta Y \Sigma I A I ' P A I A I O Y P T O T E P A I /$ ATNOTEPAI}

D'après Xénophon, il existe des autels et des temples séparés pour Ourania et pour Pandemos. Cette affirmation ne possède aucune force d'argument dans le contexte présent, étant donné que, d'une part, des temples différents existent de façon générale pour honorer les dieux sous diverses épiclèses, et que, d'autre part, Pausanias, le Périégète, apporte la preuve qu'Ourania et Pandémos peuvent coexister dans un même sanctuaire ${ }^{62}$. Abordant le rituel, le philosophe attribue à Pandémos des $\theta v \sigma i ́ a \iota$

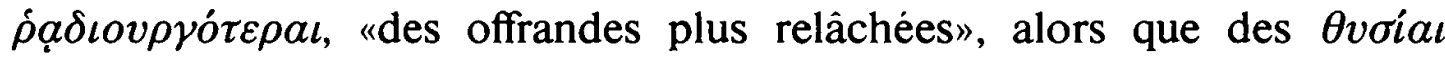

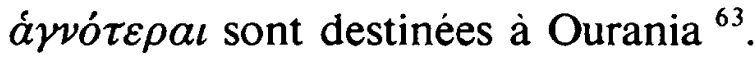

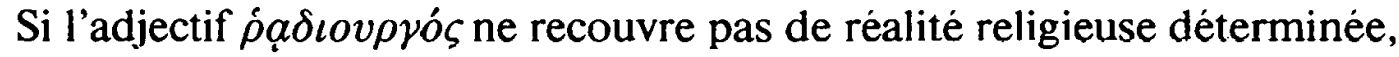
l'épithète á $\gamma v o ́ s$, par contre, nous introduit directement dans le domaine du sacré et permet la comparaison avec un autre témoignage ayant trait au culte d'Ourania à Athènes. Voici, en effet, ce que rapporte le scholiaste commentant l'CEdipe à Colone de Sophocle à propos des offrandes destinées aux Euménides :

Car on ne répand pas sur elles du vin, mais de l'eau, et leurs libations sont appelées sobres ( $m \varphi a ́ d \iota a \imath)$. Polémon, dans le Contre Timée, écrit comme suit que des sacrifices à certains autres dieux aussi sont sobres : "car les

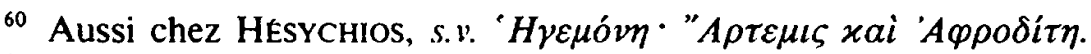

${ }^{61}$ Ainsi, on voit qu'Apollodore ( 244 F $113 \mathrm{~J}$.) s'appuie sur une localisation erronée de l'ancienne agora, à moins qu'il n'ait confondu deux sanctuaires de la deesse, pour expliquer l'épithète d'Aphrodite, preuve qu'à basse époque, la localisation du culte sur le flanc de l'Acropole pouvait poser probleme.

${ }^{62}$ Cf. supra, n. 20.

${ }^{63}$ XENOPHON, Banquet, 8, 9-10. 
Athéniens, ètant attentifs à ce genre de choses et respectueux de ce qui touche aux dieux, consacrent des offrandes sobres à la Muse Mnémosyne, à Éos, à Hélios, à Séléné, aux Nymphes, à Aphrodite Ourania»" ${ }^{64}$.

Les $\nu \eta \varphi a ́ \lambda \iota a$ sont principalement des offrandes d'eau et de miel, par opposition à l'utilisation de vin ${ }^{65}$. Ainsi, dans son ouvrage Sur la Piété, Théophraste fait remonter cet usage très haut dans le temps, les libations étant l'apanage des peuples primitifs ${ }^{66}$. Empédocle avait déjà émis des considérations dans le même sens, en des termes d'autant plus intéressants pour notre propos qu'ils touchent au culte d'Aphrodite :

Chez eux (les peuples de l'Âge d'or), ni Arès ni le Tumulte ne comptaient au nombre des dieux. Zeus ne régnait pas, ni Cronos, ni Poséidon, mais Cypris était reine. Ils se la rendaient favorable par de pieuses offrandes, des peintures d'animaux, des parfums aux mille senteurs, des sacrifices de myrrhe pure et d'encens odorant, répandant sur le sol les libations des abeilles d'or. Et l'autel n'était point arrosé par le sang généreux des taureaux, et il n'y avait pire souillure chez les hommes que d'en manger les membres après leur avoir arraché la vie ${ }^{67}$.

Sans entrer dans le détail d'une analyse qui n'a pas sa place ici, on peut dire que le passage de l'offrande végetale au sacrifice animal sanglant est considéré comme une perversion, une chute de l'humanité, conférant ainsi une sacralité plus grande aux $\nu \eta \varphi a ́ \lambda \iota a$, signe tangible de l'innocence primitive.

Dans ce contexte, rappelons la signification de áyvós et essayons d'en expliquer l'emploi par Xénophon. Selon J. Rudhardt, árvós désigne une qualité éminemment respectable, un caractère élevé et imposant. Il signifie une sorte de "pureté extratemporelle» ${ }^{68}$, définition on ne peut mieux

${ }^{64}$ Polemon, frg. 42 Müller ( $F H G$, III, p. 127), cité par schol. Sophocle, Edipe $a$

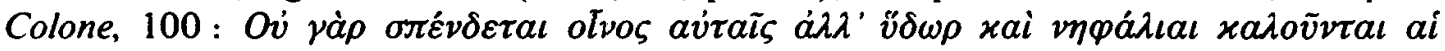

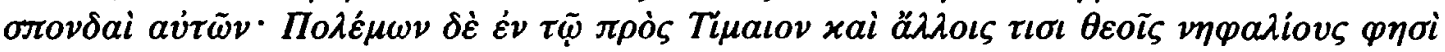

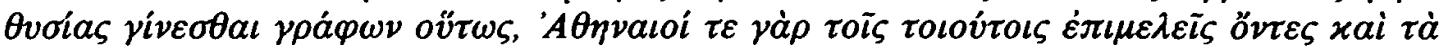

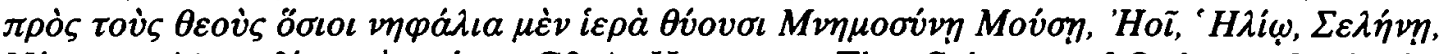

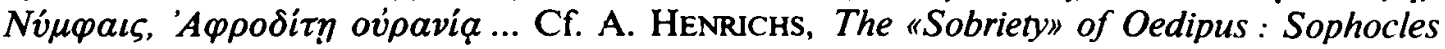
OC 100 Misunderstood, dans HSPh, 87 (1983), pp. 87-100.

65 P. STENGEL, Opferbräuche der Griechen, Leipzig, 1910, pp. 180-181; L. ZIEHEN, art. Niqúd $\iota$, dans RE, XVI, 2 (1935), col. 2481-2489.

${ }_{66}$ PORPHYRE, De l'abstinence, II, 20, 2-3.

${ }^{67}$ Empédocle, frg. 128 Diels-Kranz ${ }^{6}$, cité par PorPhyre, II, 21, 2-3; 27, 7 (trad. J. Bouffartigue).

${ }^{68}$ J. RUDHARDT, o.l., pp. 39-41. 
adaptée, d'après ce qui a été dit, aux $v \eta \varphi a ́ \lambda \iota a$, les offrandes sobres de Polémon.

Il semble donc que l'opposition rituelle entre Aphrodite Pandémos et Ourania repose sur la différence entre sacrifices sanglants et offrandes non

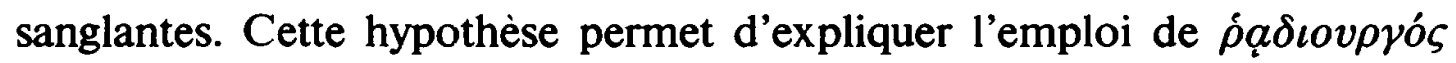
qui vise sans doute à sanctionner la perversion de la pratique, donc de l'homme. Reste à voir s'il existe une commune mesure entre cette interprétation possible du texte de Xénophon et les réalités du culte.

Les offrandes non sanglantes s'adressent généralement aux morts et aux divinités chthoniennes et l'on utilise le mot xoaí de préférence au mot $\sigma \pi o v \delta a i^{69}$. Si on reprend le témoignage de Polémon, il s'avère que Mnémosyne et Hélios reçoivent des $\nu \eta \varphi a ́ \lambda \iota a$, comme l'atteste par ailleurs une inscription du rv $v^{e}$ siècle avant J.-C. ${ }^{70}$. Plutarque nous apprend aussi que les Muses reçoivent ce genre d'offrandes ${ }^{71}$. Quant à Éos et Séléné, elles sont apparemment liées à Hélios et forment un groupe ${ }^{72}$. D'après $P$. Stengel, les Nymphes doivent à leur caractère chthonien d'être ainsi honorees ${ }^{73}$.

En ce qui concerne Aphrodite Ourania, les parallèles sont difficiles à trouver, et le texte d'Empédocle illustre le règne d'Aphrodite sur une monde désincarné, un âge d'or qu'il est délicat de comparer à l'époque historique, d'autant plus que Lucien, dans un passage déjà retenu à plusieurs reprises ${ }^{74}$, semble indiquer que des animaux étaient bel et bien sacrifiés à Ourania. La seule explication du texte de Polémon réside dans l'absence de vin pour les libations en l'honneur de la déesse. Mais, dans

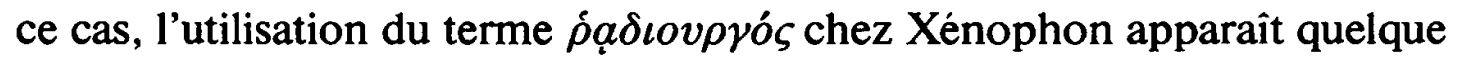
peu démesurée et cette seule différence entre les deux cultes, libations avec vin d'un côté et abstention de l'autre, justifie assez faiblement une séparation aussi stricte, voire une réelle opposition.

Au terme de cette étude, il apparait donc clairement que, s'il existe bien des différences entre les deux cultes, elles ne justifient en aucun cas l'opposition irréductible entre Ourania et Pandémos. Malgré le succès que la conception intellectuelle a connu dans son interpretation plus populaire

${ }^{69}$ Ibid., pp. 246-247.

70 I.G., $\mathrm{II}^{2}, 4962$ = DITTENBERGER, Syll. ${ }^{3}, 1040$ : il s'agit d'une loi sacree découverte au Piree. Cf. AthEnEE, XV, 693e.

${ }^{71}$ Plut., Moralia, 156d.

72 P. Stengel, o.l., p. 181.

73 Ibid.

${ }^{74}$ LuCien, Dial. Court., 7, 1. 
- qu'elle lui soit parallèle ou qu'elle en soit dérivée -, les deux épiclèses de la déesse ne la divisaient pas en figures divines aussi antagonistes. Et les courtisanes, qui ont sans doute quelque peu altéré la majesté divine de la déesse en l'érigeant en patronne de leur "corporation", adressaient autant leurs vœux à Ourania qu'à Pandémos. Inversement, la dignité attribuée au culte d'Ourania est tout aussi présente dans celui de Pandémos.

On le voit, la marge est grande entre le niveau moral des conceptions religieuses quand elles servent une réflexion qui prétend se fonder sur la mythologie ou sur les rites, et les attributions réelles accordées aux différentes épithètes des divinités. Et lorsque Sophocle déclare que «Cypris n'est pas seulement Cypris, elle porte encore beaucoup d'autres noms» ${ }^{75}$, il ne scinde pas la déesse en entités irréductibles, mais vise à rendre compte de la multiplicité de ses pouvoirs. En somme, Cypris n'est pas seulement Cypris, mais elle reste toujours Cypris.

Rue André Renard 10, (novembre 1986) B-4624 Romsée. Vinciane PirenNe-Delforge.

${ }^{75}$ SoPHOClE, frg. 855 Nauck $^{2}$, cité par StobEE, Florilège, 63, 6. 